\title{
CYCLIC RELATIONS IN POINT SET THEORY*
}

\author{
BY E. C. STOPHER, JR.
}

1. Introduction. The formula

$$
\phi c \phi c \phi c \phi A=\phi c \phi A,
$$

where $c$ denotes the operation of taking complements and $\phi$ is an arbitrary operator, is of considerable interest in the study of sets of points. Kuratowski $\dagger$ proved that the formula holds for a postulated closure or extension function. Zarycki $\ddagger$ established the formula in case $\phi A$ is the "interior" of $A$ and Sandersई established it for a general derived set operator satisfying the postulates:

I.

II.

$$
\begin{aligned}
d(A+B) & =d A+d B, \\
d^{2} A & \leqq d A .
\end{aligned}
$$

In this paper we shall follow the established $\|$ practice of postulating a derived set operator $d$, subject to I and II, and, using a notation introduced by Chittenden, define certain terms as follows:

Identity:

$$
1 A=A
$$

Complement: $c A=S-A(S$ denotes the entire space);

Extension: $\uparrow$

$$
e A=A+\ddot{d} A ;
$$

* Presented to the Society, April 10, 1937.

† C. Kuratowski, Sur l'opération Ā de l'analysis situs, Fundamenta Mathematicae, vol. 3 (1922), pp. 182-199.

$\ddagger$ M. Zarycki, Notions fondamentales de l'analysis situs, Fundamenta Mathematicae, vol. 9 (1927), pp. 3-15.

$\S \mathrm{S}$. T. Sanders, Jr., Derived sets and their complements, this Bulletin, vol. 42 (1936), pp. 577-584.

|| F. Riesz, Stetigkeitsbegriff und abstrakte Mengenlehre, Atti del 4 Congresso Internationale dei Matematici, Roma, 1910, vol. 2, p. 18; Chittenden, On general topology and the relation of the properties of the class of all continuous functions to the properties of space, Transactions of this Society, vol. 31 (1929), pp. 290-321.

IF. Hausdorff, Mengenlehre, pp. 109-129. Under the postulates given, the derived set corresponds to Hausdorff's set of $\beta$ points, $A_{\beta}$. The extension corresponds to his $\alpha$ points, $A_{\alpha}$. Similarly, $h A$ corresponds to $A_{h}, j A$ to $A_{j}, b A$ to $A_{r}$ (border is a translation of the German word "rand"), $k A$ to $A_{k}$, and $s A$ to $A_{s}$. 


$\begin{array}{ll}\text { Interior: } & i A=A c d c A ; \\ \text { Concentrated part: } & h A=A d A ; \\ \text { Isolated part: } & j A=A c d A ; \\ \text { Border: } & b A=A d c A ; \\ \text { Frontier: } & f A=A d c A+c A d A ; \\ \text { Kernel: } & k A=\sum B \leqq A, \text { such that } B \leqq d B ; \\ \text { Separated part: } & s A=A c k A .\end{array}$

In $\S \S 2-7$ it will be shown that each of these operators except $b$ and $h$ satisfies the Kuratowski formula. It might be pointed out that these operators do not all have the same basic properties. In contrast to Postulates I and II we have, for example,

$$
\begin{aligned}
i(A+B) & \geqq i A+i B, \\
i(A B) & =i A i B, \\
i^{2} A & =i A, \\
f(A+B) & \leqq f A+f B, \\
f^{2} A & \leqq f A .
\end{aligned}
$$

This list of operators is by no means a complete list of operators satisfying the Kuratowski formula, as can be readily seen by considering the formula

$$
\psi^{4} A=\psi^{2} A .
$$

We have the proposition that if an operator $\theta$ satisfies formula (1) $((2))$, its transform* satisfies (1) ((2)) and its complement satisfies (2) ((1)). We make use of this proposition in $\S 8$ to obtain additional operators satisfying the Kuratowski formula.

Examples will be given in $\S 9$ to show that the $b$ and $h$ operators do not in general satisfy the Kuratowski formula. It will be shown in $\$ \S 10$ and 11 that each of $(b c)^{\beta} b A$ and $(h c)^{\beta} h A$ with increasing $\beta$ defines a set.

2. Identity, Complement. The identity operator can be used in the Kuratowski formula since, on account of the relation $c^{2} A=A$, each side of the equation reduces to $c A$. It is readily seen also that the complementary operator can be used in place of $\phi$, each side of the equation again reducing to $c A$.

* An operator $\phi$ is said to be the transform of an operator $\psi$, if $\phi A=c \psi c A$; it is the complement of the operator $\psi$, if $\phi A=c \psi A$. 
3. Extension, Interior. The general "defined" extension function satisfies the Kuratowski formula since this function satisfies Kuratowski's postulates I, II, and IV, which Kuratowski* showed were sufficient. We immediately have

$$
\text { iciciciA }=i c i A \text {, }
$$

since the interior and extension operators are transforms of each other.

4. Frontier. Since $f c A=f A, f^{2} A=b f A$, and $b^{2} A=b A$, both $f c f c f c f A$ and $f c f A$ reduce to $b f A$, establishing the formula

$$
f c f c f c f A=f c f A \text {. }
$$

5. Isolated Part. By definition,

$$
\begin{aligned}
j A= & A c d A \\
c j A= & c A+h A \\
j c j A= & (c A+h A) c d c A c d h A \\
= & c A c d c A c d h A(c d j A+d j A)+h A c d h A c d c A \\
= & c A c d c A c d h A c d j A+c A c d c A c d h A d j A \\
& +A d A c d h A c d c A \\
= & c A c d(c A+h A+j A)+c A c d c A c d h A d j A \\
& +A d j A c d h A c d c A \\
= & c A c d S+d j A c d h A c d c A(c A+A) \\
= & J c A+j d S c d c A . \dagger
\end{aligned}
$$

Replacing $A$ by $c j A$, we have

$$
\begin{aligned}
j c j c j A & =J j A+j d S c d j A \\
& =J A+j d S c d A,
\end{aligned}
$$

since $c d A=c d j A c d h A$ and $c d h A$ includes $j d S$. Again replacing $A$ by $c j A$, we obtain

$$
\begin{aligned}
j c j c j c j A & =J c j A+j d S c d c j A \\
& =J c A+J h A+j d S c d c A c d h A \\
& =J c A+j d S c d c A,
\end{aligned}
$$

* C. Kuratowski, loc. cit.

$\dagger J=c d S$. Symbol used by Sanders, loc. cit. 
again because $c d h A$ includes $c d^{2} S$, which in turn includes $j d S$. This establishes the formula

$$
j c j c j c j A=j c j A .
$$

6. Separated Part. By definition,

$$
\begin{aligned}
s A & =A c k A, \\
c s A & =c A+k A, \\
s c s A & =(c A+k A) c k c s A .
\end{aligned}
$$

To evaluate the set $k \operatorname{cs} A$ we make use of the fact that for any set $B, k B$ is equal to the limit as $\beta$ increases of $h^{\beta} B$. By definition,

$$
\begin{aligned}
h c s A & =(c A+k A)(d c A+d k A) \\
& =h c A+d k A,
\end{aligned}
$$

since the product of $s A$ and $d k A$ is null; and

$$
\begin{aligned}
h^{2} c s A & =(h c A+d k A)(d h c A+d k A) \\
& =h^{2} c A+d k A,
\end{aligned}
$$

$d k A$ being perfect, that is, $d^{2} k A=d k A$. Continuing, we see that

Hence,

$$
\begin{aligned}
h^{\beta} c s A & =h^{\beta} c A+d k A, \\
k c s A & =k c A+d k A=c s A k S .
\end{aligned}
$$

$$
\begin{aligned}
c k c s A & =s A+s S, \\
s c s A & =(c A+k A)(s A+s S) \\
& =c A s S,
\end{aligned}
$$

$k A$ being a subset of $k S$ and not of $s S$.

Substituting $\operatorname{cs} A$ for $A$, we have

$$
\operatorname{scscs} A=s A s S=A s S .
$$

Again substituting $c s A$ for $A$, we obtain

$$
\begin{aligned}
\operatorname{scscscs} A & =c s A s S=c A s S+k A s S \\
& =c A s S,
\end{aligned}
$$

establishing the formula

$$
\operatorname{scscscs} A=\operatorname{scs} A .
$$


7. Kernel. By definition,

$$
\begin{aligned}
k A & =A c s A, \\
c k A & =c A+s A, \\
k c k A & =k c A+s A d k c A .
\end{aligned}
$$

Substituting $c k A$ for $A$, we have

$$
\begin{aligned}
k c k c k A & =k^{2} A+s c k A d k^{2} A \\
& =k A+s c k A d k A .
\end{aligned}
$$

We observe that

$$
\begin{aligned}
s c k A & =c k A c k c k A \\
& =(c A+s A) c k c A(c s A+c d k c A) \\
& =s c A+s A c d k c A \\
& =s c A+A s S,
\end{aligned}
$$

since $s A c d k c A k S=0$. Therefore,

$$
\begin{aligned}
k c k c k A & =k A+(s c A+A s S) d k A \\
& =k A+s c A d k A .
\end{aligned}
$$

Again substituting $c k A$ for $A$, we have

$$
\begin{aligned}
k c k c k c k A & =k c k A+s k A d k c k A \\
& =k c k A,
\end{aligned}
$$

since $s k A=0$.

8. Additional Operators. Since the transforms of operators satisfying the Kuratowski formula also satisfy it, we immediately obtain the fact that the following operators satisfy the Kuratowski formula: $c d c, c j c=(1+h c), c f c=(i+i c), c k c=(1+s c)$, and $c s c=(1+k c)$.

Since $e, i, j, b, f, k$, and $s$ are known to satisfy equation (2), it follows immediately that $i c,(c+d c),(c+h),(c+i),(i+i c)$, $(c+s)$, and $(c+k)$ satisfy the Kuratowski formula.

9. Examples. Equation (1) will not hold in general for $\phi$ equal to either of the remaining two operators, $b$ and $h$, as the following examples show. 
Let the space $S$ be the closed linear interval $(0,1)$. Let the set $A$ be the points $(1 / 2,3 / 4,7 / 8, \cdots)$. Then,

$$
\begin{aligned}
b A & =A, \\
b c b A & =1, \\
b c b c b A & =\text { null, } \\
b c b c b c b A & =\text { null } \\
& \neq b c b A .
\end{aligned}
$$

For the second example, consider the same space $S$ but include also the point 1 in the $\operatorname{set} A$. Then,

$$
\begin{aligned}
h A & =1, \\
h c h A & =S-1, \\
h c h c h A & =\text { null, } \\
h c h c h c h A & =S \\
& \neq h c h A .
\end{aligned}
$$

Although equation (1) does not hold for either $b$ or $h$, it is interesting to note in these examples that

$$
\begin{aligned}
b c b c b c b c b A & =b c b c b A, \\
h c h c h c h c h A & =h c h c h A .
\end{aligned}
$$

10. Border. Examining further the operator $b$, we see that

$$
c b A=c A+i A,
$$

and

$$
\begin{aligned}
b c b A & =(c A+i A) d b A \\
& =b c A d b A,
\end{aligned}
$$

since $d b A$ is included in $f A$ rather than in $i A$.

Substituting $c b A$ for $A$ and making use of the fact that $c^{2}=1$ and $b^{2}=b$, we have

$$
\begin{aligned}
b c b c b A & =b c c b A d b c b A \\
& =b A d b c b A \\
& \leqq b A .
\end{aligned}
$$

Thus we see that we have two monotonic decreasing se- 
quences of sets, $(b c)^{2 \beta} b A$ and $(b c)^{2 \beta+1} b A, \beta=1,2,3, \cdots$. Chittenden has pointed out that, since a set is determined by every product $\Pi A_{\beta},(\beta=1,2,3, \cdots)$, where $\left[A_{\beta}\right]$ represents a monotonic decreasing sequence of sets, each of these sequences defines a set and we have implied that for all ordinals $\alpha$ greater than or equal to some finite or transfinite ordinal $\alpha_{0}$,

$$
(b c)^{\alpha+2} b A=(b c)^{\alpha} b A \text {. }
$$

11. Concentrated Part. Similarly, it can be shown that, with increasing $\beta,(h c)^{2 \beta} h A$ defines a set. We have

$$
\begin{aligned}
h A & =A c j A, \\
c h A & =c A+j A, \\
h c h A & =(c A+j A)(d c A+d j A) \\
& =h c A+j A d c A+c A d j A .
\end{aligned}
$$

Substituting $\operatorname{ch} A$ for $A$, we may write,

$$
(h c)^{2} h A=h^{2} A+j c h A d h A+h A d j c h A \text {. }
$$

However,

$$
\begin{aligned}
j c h A= & (c A+j A) c d c A c d j A \\
= & c A c d c A c d j A c d h A+c A c d c A c d j A d h A \\
& +j A c d c A,
\end{aligned}
$$

$j A$ being included in $c d j A$. This simplifies to

and we have

$$
\begin{aligned}
j c h A= & c A c d(c A+j A+h A)+j c A c d j c A c d j A d h A \\
& +A c d A c d c A \\
= & J c A+J A+j c A i d S \\
= & J+j c A i d S,
\end{aligned}
$$

$$
\begin{aligned}
(h c)^{2} h A & =h^{2} A+(J+j c A i d S) d h A+h A(d J+\overline{j c A i d S)} \\
& =k A+s^{2} A+j c A i d S+\operatorname{sh} A d J \\
& =(k A+j c A i d S) d k A+\operatorname{sh}^{2} A i d S+\operatorname{sh} A d J
\end{aligned}
$$

since $h^{2} A=k h^{2} A+\operatorname{sh}^{2} A$ and $k h A=k A$.

Again substituting $\operatorname{chch} A$ for $A$, we have

$$
\begin{aligned}
(h c)^{4} h A= & (k \operatorname{chch} A+j h c h A i d S) d k c h c h A \\
& +\operatorname{sh}^{2} \operatorname{chchA} i d S+\operatorname{shchchAdJ} .
\end{aligned}
$$


But,

$$
\begin{aligned}
k c h c h A= & k h c h c h A=k A+j c A i d S, \\
d k c h c h A= & d k A+d(j c A i d S)=d k A, \\
j h c h A i d S= & i d S(h c A+j A d c A \\
& +c A d j A) c d h c A c d(j A d c A) c d(c A d j A) \\
= & i d S[j h c A c d(c A d j A) c d(j A d c A) \\
& +j(c A d j A) c d h c A c d(j A d c A) \\
& +j(j A d c A) c d h c A c d(c A d j A)] \\
= & i d S \cdot j h c A, \\
s h^{2} c h c h A i d S= & h\left(s h^{2} A i d S\right)=\operatorname{sh}^{3} A i d S, \\
\operatorname{shchchAdJ=} & s h A d J .
\end{aligned}
$$

Making these substitutions, we obtain

$$
\begin{aligned}
(h c)^{4} h A= & (k A+j c A i d S+j h c A i d S) d k A+s^{3} A i d S \\
& +s h A d J .
\end{aligned}
$$

Continuing, we have

$$
\begin{aligned}
(h c)^{2 \beta} h A= & {\left[k A+\sum_{\nu=0}^{\beta-1} j h^{\nu} c A i d S\right] d k A } \\
& +\operatorname{sh}^{\beta+1} A i d S+\operatorname{sh} A d J
\end{aligned}
$$

Since

$$
\begin{aligned}
s c A & =j c A+j h c A+j h^{2} c A \cdots \\
& =\sum_{\nu=0} j h^{\nu} c A,
\end{aligned}
$$

and $s h^{\beta} c A=h^{\beta} s c A \rightarrow 0$, with increasing $\beta$, it is apparent that with increasing $\beta$,

$$
\begin{aligned}
(h c)^{2 \beta} h A & \rightarrow(k A+s c A i d S) d k A+s h A d J \\
& =(k A+s c A) i d S+h A d J
\end{aligned}
$$

and this establishes the proof.

$* \rightarrow$ is the ordinary symbol for convergence; $A_{\beta} \rightarrow A$, with increasing $\beta$, is equivalent to $\Pi\left(A c A_{\beta}+A_{\beta} c A\right)=0,(\beta=1,2,3, \cdots)$. 
12. Summary. The results of $\$ \S 2-7$ can be summarized in the following theorem:

THEOREM. The Kuratowski formula,

$$
\phi c \phi c \phi c \phi A=\phi c \phi A,
$$

is satisfied for $\phi$ equal to any of the operators $1, c, d, e, i, j, f, k$, and $s$.

Sections 10 and 11, together with this theorem, imply the following corollary:

Corollary. The equation

$$
(\phi c)^{\alpha+2} \phi A=(\phi c)^{\alpha} \phi A
$$

holds for every ordinal $\alpha$ equal to or greater than some finite or transfinite ordinal $\alpha_{0}$, and for $\phi$ equal to any of the operators $1, c, d$, $e, i, h, j, b, f, k$, and $s$.

UNIVERSITY OF IOWA

\title{
A CONDITION THAT A FIRST BOOLEAN FUNCTION VANISH WHENEVER A SECOND DOES NOT
}

\author{
BY J. C. C. MCKINSEY*
}

It is well known $\dagger$ that if two polynomials $f\left(x_{1}, \cdots, x_{n}\right)$ and $g\left(x_{1}, \cdots, x_{n}\right)$ in the field of complex numbers are such that $f$ vanishes whenever $g$ does not, then at least one of the two polynomials $f$ and $g$ is identically zero. The corresponding law, however, does not, in general, hold for Boolean functions, as may be seen by considering the two functions $x$ and $x^{\prime}$ in a twoelement Boolean algebra; the statement that either $x=0$ or else $x^{\prime}=0$ in a two-element Boolean algebra is, indeed, the familiar law of excluded middle. It is the purpose of the present note to determine the conditions on the coefficients of two Boolean functions in order that the first vanish whenever the second does not.

The condition found involves prime Boolean elements, which are defined as follows:

\footnotetext{
* Blumenthal Research Fellow.

$\dagger$ See, for example, Bocher, Introduction to Higher Algebra, p. 8.
} 